\title{
Phase transitions in multicomponent liquid systems: The key role of electrolytes
}

\author{
B. M. Jaffar Ali, Jaby Jacob and A. Kumar*
}

Department of Physics, Indian Institute of Science, Bangalore 560 012, India

\begin{abstract}
The light scattering measurements, that yield osmotic compressibility $\left(\chi_{T}\right)$, are presented near and away from Special Critical Points in multicomponent liquid mixtures. A double critical point (DCP) is located in the system (3-methylpyridine + water + sodium bromide). A phase boundary is generated in the proximity of Critical Inflection Point(CIP) and it is explored in the system (ethanol + water + potassium carbonate). A Plait point is probed in the system (ethanol + water + sodium carbonate). The findings away from the $\mathbf{D C P}(\mathrm{i} . \mathrm{e}$. , for larger salt concentrations) suggest an interplay between two competing length scales. A recurring theme in these results is that the presence of electrolytes induces the Mean-Field (or classical) critical behaviour in the vicinity of a CIP, a Plait point and far away from a DCP. These observations lead to the inference of a possible structuring of the host liquid mixture around the ionic solute.
\end{abstract}

\section{INTRODUCTION}

The phenomenon of single and multiply reentrant phase transitions has drawn considerable attention recently, both from theoretical and experimental standpoints[1]. It occurs in diverse condensed and soft-condensed matter systems, e.g., ternary (or quaternary) liquid mixtures, binary gases, microemulsions, gels, dilute suspensions of charge-stabilizied colloids, granular superconductors, adsorbed monolayers, etc.[1]. However, the above phenomenon can be studied most quantitatively in multicomponent liquid mixtures. The limit of the vanishing reentrance in these mixtures defines unique thermodynamic states (or Special Critical Points), e.g., a double critical point (DCP), a critical double point (CDP), a quadruple critical point (QCP), a critical inflection point (CIP), etc.[1]. At DCP, a closed loop phase diagram (bounded by an upper and lower critical solution temperatures, $\mathrm{T}_{U}$ and $\mathrm{T}_{L}$, respectively) shrinks to a point, signifying the disappearance of reentrant miscible (or homogeneous) phases. A CDP is analogous to DCP except that the reentrant phases are the immiscible ones. A CIP denotes the merger of a DCP with its conjugate CDP and it has not yet been observed in any system. A QCP emerges when two independent DCPs merge in an appropriate field space.

In order to test lattice-solution as well as phenomenological models of reentrant miscibility, one needs to approach these Special Critical Points very closely. A common method is to vary the applied pressure in binary or ternary liquid mixtures. An alternate way is to add additional chemical components [isotopic/isomeric substitution/ salts (or electrolytes)]. The latter method has led to an incredibly close approach to DCP (3-methylpyridine + water + heavy water; 3-methylpyridine + water + sodium chloride)[1], CDP (polystyrene + acetone + water) [2], QCP (3-methylpyridine + water + heavy water + potas-

"Corresponding author 
sium iodide)[3]. The electrolytes modify the phase behaviour of the host (binary or ternary) liquid mixtures in a sensitive way. For instance, addition of $\sim 0.1$ weight $\%$ of sodium chloride to a normally miscible mixture of (3-methylpyridine + water) causes the appearance of a closed loop phase diagram[1]. This salt reduces the strength of hydrogen bonding between 3-methylpyridine and water, inhibiting the reentrance of miscible phases. At QCP, several thermodynamic excess functions such as excess volume $\left(\mathrm{v}^{E}\right)$, excess enthalpy $\left(\mathrm{h}^{E}\right)$ and excess entropy $\left(\mathrm{s}^{E}\right)$ ] go to zero simultaneously - a phenomenon induced by potassium iodide in (3-methylpyridine + water + heavy water) $[3,4]$. Electrolytes are, thus, indispensable in realizing several Special Critical Points.

In this article, we assess the role of electrolytes from the standpoint of the critical behaviour in the neighbourhood of a DCP, a CIP and a Plait point. The experimental techniques employed are: laser light scattering, turbidity and the visual observations. The systems selected are: (3-methylpyridine + water + sodium bromide) for studies concerning crossover behaviour, (ethanol + water + potassium carbonate) for investigations regarding a CIP and (ethanol + water + sodium carbonate) for probing a Plait point.

\section{EXPERIMENTAL}

We have measured the intensity of scattered light at $90^{\circ}$. The measured scattered intensity data were corrected for sample turbidity, background contributions, and incident laser intensity fluctuations. This corrected intensity, denoted by $\mathrm{I}_{s}$, can be adequately described $[1,3]$ by the following expression:

$$
\frac{I_{s}(q)}{T}=I_{o} \frac{\pi^{2}}{\lambda_{o}^{4}}\left(\frac{\partial n^{2}}{\partial c}\right)^{2} k_{B} \chi_{T}\left(1+q^{2} \xi^{2}\right)^{-1+\eta / 2}
$$

where $I_{0}$ is the incident laser intensity, $\lambda$ is the vacuum wave length of incident light $(=632.8 \mathrm{~nm}), \mathrm{n}$ is the concentration (c) dependent refractive index, $\chi_{T}$ is the osmotic susceptibility, $q\left[=\frac{4 \pi n}{\lambda} \sin \left(\frac{\theta}{2}\right), \theta\right.$ is the scattering angle, equal to $90^{\circ}$ in this case] is the scattering wave vector, $\xi$ is the correlation length of concentration fluctuations, and $\eta$ is the Green-Fisher exponent. Near the critical point, $\chi_{T}$ and $\xi$ exhibit the following scaling forms:

$$
\begin{aligned}
\chi_{T} & =\chi_{0} t^{-\gamma}\left(1+\chi_{1} t^{\Delta_{1}}+\chi_{2} t^{2 \Delta_{1}}+\cdots\right) \\
\xi & =\xi_{0} t^{-\nu}\left(1+\xi_{1} t^{\Delta_{1}}+\xi_{2} t^{2 \Delta_{1}}+\cdots\right)
\end{aligned}
$$

where $t$ is the reduced temperature $\left[=\left(\mathrm{T}_{c}-\mathrm{T}\right) / \mathrm{T}_{c}, \mathrm{~T}_{c}\right.$ is $\mathrm{T}_{U}$ or $\left.\mathrm{T}_{L}\right], \gamma$ and $\nu$ are the critical exponents; $\chi_{0}$ and $\xi_{0}$ are the critical amplitudes; $\chi_{1}, \chi_{2} \ldots$ etc., are the correction-to-scaling amplitudes of $\chi_{T}, ; \xi_{1}, \xi_{2} \ldots$, etc., are the correction-toscaling amplitudes of $\xi$ and $\Delta_{1}(\simeq 0.5)$ is the leading correction-to-scaling exponent. Near an Ising critical point, $\gamma \simeq 1.24, \nu \simeq 0.63$, and $\eta \simeq 0.03[1]$.

\section{A. CROSSOVER BETWEEN ISING-LIKE AND MEAN-FIELD CRITICAL BEHAVIOUR}

We examine the nature of critical behaviour in a solvophobic system with varying amounts of electrolyte. The system chosen for these studies is a ternary liquid mixture of [3-methylpyridine (MP) + water $(\mathrm{W})+$ sodium bromide $(\mathrm{NaBr})$ ] The binary liquid mixture of $(\mathrm{MP}+\mathrm{W})$ is miscible at all temperatures at normal pressure. But a closed loop of size $\Delta \mathrm{T}\left[\Delta \mathrm{T}=\left(\mathrm{T}_{U^{-}}-\mathrm{T}_{L}\right)\right]$ appears with the addition of a small amount of salt (e.g., $\mathrm{NaCl}, \mathrm{KBr}, \mathrm{NaBr}$, etc). For all the salts mentioned above the loop size $(\Delta \mathrm{T})$ increases $\left(\mathrm{T}_{L}\right.$ decreases and $\mathrm{T}_{U}$ increases $)$ with increasing salt concentration. The rate of change of $T_{L} \mathrm{~s}$ and $\mathrm{T}_{U} \mathrm{~s}$ are the least in the case of $\mathrm{NaBr}$ compared to the other salts. Hence, experiments can be performed over a wider salt concentration in this system as compared to the 
other salts. For $\mathrm{NaCl}$, the DCP occurs at a temperature of approximately $77^{\circ} \mathrm{C}$ and for $0.097 \mathrm{wt}$. \% of $\mathrm{NaCl}[1]$. In the case of $\mathrm{NaBr}$, the coordinates of DCP are $\simeq 80^{\circ} \mathrm{C}$ and 0.45 wt. \% of $\mathrm{NaBr}$. The line of critical points is determined visually (Fig.1). The extremum of this line defines a DCP. Samples are adjusted for criticality with respect to $\mathrm{T}_{L}$ only. The critical value of MP concentration was determined using the equal volume coexistence criterion. The critical concentration of MP, $\mathrm{x}_{M P}$ [wt. of MP in wt. of $(\mathrm{MP}+\mathrm{W})]$ is $\simeq 0.31$ and it decreases gradually with the increasing salt concentration. $\mathrm{T}_{L}$ s are detected to within $\pm 10 \mathrm{mK}$ and $T_{U} S$ are located to within $\pm 50 \mathrm{mK}$ in most of the cases. $\mathrm{T}_{U} \mathrm{~s}$ above $140^{\circ} \mathrm{C}$ were not determined since the boiling point of

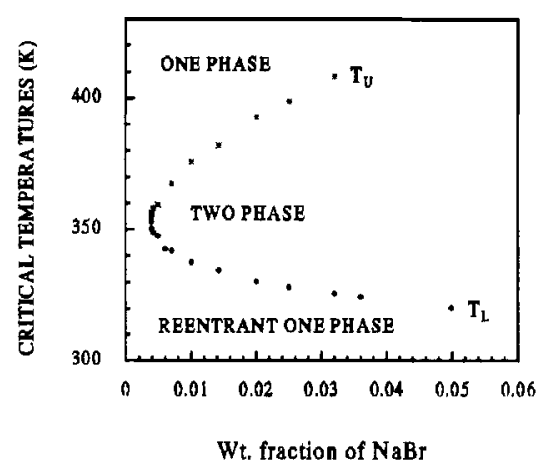

Fig. 1. Plot of Upper and Lower Critical Solution Temperatores ( $T_{U}$ and $T_{L}$, respectively) versus $X_{\mathrm{N}_{1 B}}$ (weight fraction of $\mathrm{NaBr}$ ) for the system: 3-methylpyridine + water + $\mathrm{NaBr}$. the mixture is close to $140^{\circ} \mathrm{C}$. The line of critical points is somewhat asymmetric with respect to the DCP (Fig.1). The rate of change of $T_{U}$ is much faster as compared to that of $T_{L}$, with salt concentration (Fig.1). The light scattering data $\left(I_{s} / T\right)$ for two representative samples are shown in Fig. 2. Details of the two samples are provided in Table I. In the case of sample A, recovery of exact Ising exponents $(\gamma=1.24)$ is possible only with the use of the field variable $t_{U L}\left[t_{U L}=\left|\left(\mathrm{T}_{U}-\mathrm{T}\right)\left(\mathrm{T}_{L}-\mathrm{T}\right)\right| / \mathrm{T}_{U} \mathrm{~T}_{L}\right]$ in lieu of $t$.

Table I. Details of the samples on which light scattering measurements are performed. $\mathrm{X}_{\mathrm{NaB} r}$ is the weight fraction of $\mathrm{NaBr}$ in the total mixture.

\begin{tabular}{|l|l|l|l|l|l|}
\hline Sample & $\mathrm{X}_{N a B r}$ & $\mathbf{x}_{M P}$ & $\mathbf{T}_{L}(\mathrm{~K})$ & $\mathrm{T}_{U}(\mathrm{~K})$ & $\Delta \mathrm{T}(\mathrm{K})$ \\
\hline $\mathrm{A}$ & 0.02495 & 0.31063 & 327.594 & 398.694 & 71.1 \\
$\mathrm{~B}$ & 0.07995 & 0.30332 & 315.115 & above 414 & - \\
\hline
\end{tabular}

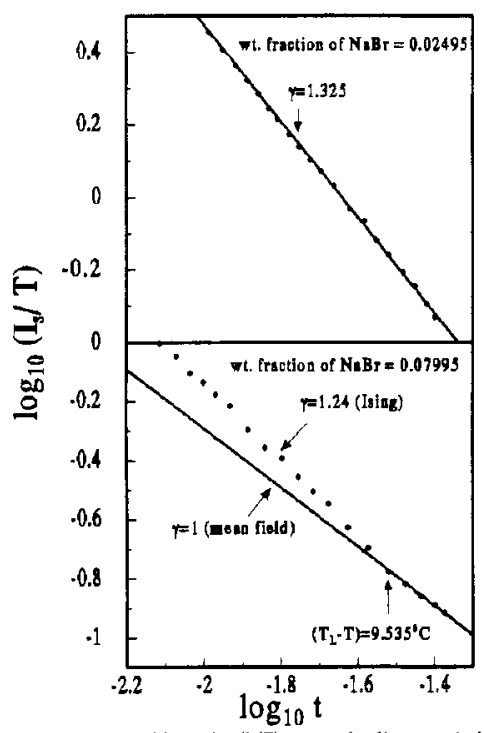

Fig. 2. Plot of acattered iateneity $(L / T)$ versus the distuoce $t|=|\left(T_{e} \cdot T\right) / T_{e} \mid$ from the lower consolute point $\left(T_{t}=T_{1}\right)$ for a ternary liquid mixtare [ 3 - methylpyridine + water + salt (sodium bromide)]. [o coserast to the case with 0.02495 weight fraction of salt, a departire from Ising behaviou ( far away from $T_{1}$ ) is clearly visible for the case with 0.07995 weirht frection of sait.
The value of $\gamma(=1.325)$ is indicated in Fig.1. This higher value of $\gamma$ (compared to the Ising value of 1.24 ) is because of the fact that field variable $t$ is used instead of $t_{U L}[1,2]$. This larger value of $\gamma$ is also due to the fact that this system is closer to a DCP $(\Delta \mathrm{T}=$ $\left.71.1{ }^{\circ} \mathrm{C}\right)$. Thus, the influence of $\mathrm{T}_{U}$ is felt, though the experimental path approaches the conjugate $\mathrm{T}_{L}$ only. For sample $\mathrm{B}$, recourse to field variable $t_{U L}$ is not required as $\Delta \mathrm{T}$ is larger compared to the one for sample $A[1]$. But a clear departure from pure Ising behaviour can be observed far away from $T_{L}$ in this case (Fig.2). We consider this departure as a signature of the mean-field critical behaviour. With the aid of Eq. (2) and (3), 
$\mathrm{I}_{s} / \mathrm{T}$ upto two correction terms is given by the Eq.(4); where, $A_{1}=$ $\left(\mathrm{I}_{0} \pi^{2} / \lambda_{0}^{4}\right)\left(\partial n^{2} / \partial c\right)^{2} \mathrm{k}_{B} \chi_{0}, \mathrm{~A}_{2}=\chi_{1}$ $\mathrm{A}_{3}=\chi_{2}$, and $\mathrm{A}_{4}=\mathrm{q}^{2} \xi_{0}^{2}$.

The data were fitted using a non-linear-least-squares procedure [5].

$$
\frac{I_{s}}{T}=\frac{A_{1} t^{-1.24}\left(1+A_{2} t^{0.5}+A_{3} t\right)}{\left\{1+A_{4} t^{-1.26}\left[1+1.3 A_{2} t^{0.5}+\left(0.65^{2} A_{1}{ }^{2}+2.2 A_{3}\right) t\right]\right\}^{0.985}}
$$

In the fit, the critical exponents are fixed at their Ising values $(\gamma=1.24, \nu=0.63$ and $\Delta_{1}=0.5$ ). Moreover, the ratio of correction-to-scaling amplitudes is also fixed at their universal values $\left(\xi_{1} / \chi_{1} \simeq 0.65\right.$ and $\left.\xi_{2} / \chi_{2} \simeq 1.1\right) . \quad \chi_{\nu}{ }^{2}$ values close to 1 (indicating a good fit) are obtained only for the fits done with two correction-toscaling terms. Results for sample $B$ with two correction terms are $A_{1}=2.83 \times 10^{-3}$, $\mathrm{A}_{2}=-2.67, \mathrm{~A}_{3}=9.762$ and $\mathrm{A}_{4}=1.038 \times 10^{-5}$ and $\chi_{\nu}{ }^{2}=1.249$.

\section{B. SEARCH FOR A CRITICAL INFLECTION POINT}

We explore a Critical Inflection Point (CIP) in a ternary liquid mixture [ethanol(E) + water $(\mathrm{W})+$ potassium carbonate][6]. The interesting feature in this system is the existence of multiple phase transitions (multiply reentrant phases) along the constant-salt axis (for a fixed $\mathbf{E} / \mathbf{W}$ ratio) at atmospheric pressure[6]. As the temperature is increased, it undergoes [2phase-1phase2phase-1phase] transitions. Thus it has double reentrant transitions. By a suitable choice of the additional component and/or pressure, the coexisting phase boundary can be made critical. In that situation, the region of multiple transitions is bounded by a Critical Double Point (CDP) and Double Critical Point (DCP). This fact motivated us to investigate the critical phenomena in this system in the vicinity of the multiple transition points as a function salt and $\mathbf{E} / \mathbf{W}$ ratio. Our goal was to explore the possibility of realization of Critical Inflection Point (CIP), wherein a DCP and a CDP merge[1].

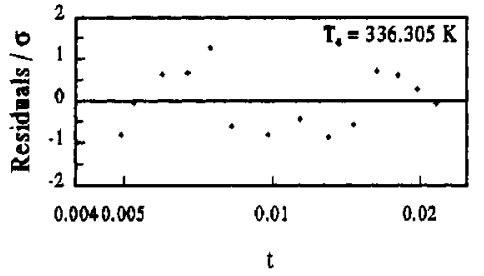

Fig. 4. Residuals of the fit to Eq. (5) for the data shown in Fig. 3.

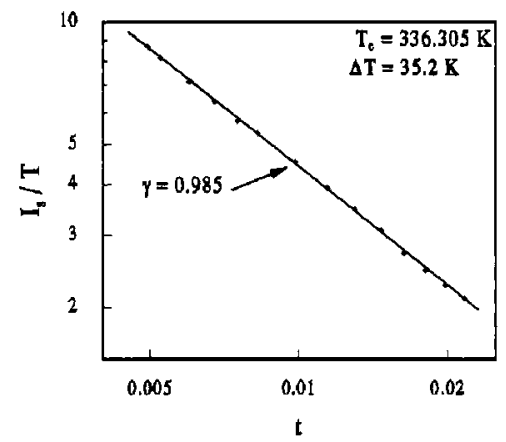

Fig. 3. Plot of scattered intensity $(\mathbf{I} / \mathrm{T})$ versus distance $t\left[=\left(T_{U}-T\right) / T_{U} l\right]$ for an approsech to the convex phase boundary in a ternary liquid mixture [Ethanol + Water $\left.+\mathrm{K}_{2} \mathrm{CO}_{3}\right]$. Solid line is a least square fit to the experimental points as described in the text.

As a first step towards this goal, we have characterized the coexistence surface near the region of double reentrance. The multiple transitions occur at around $13.55 \mathrm{wt} . \%$ of salt in the total mixture. This concentration is different for different $\mathbf{E} / \mathrm{W}$ ratios. The region wherein measurements can be performed exist within

a very narrow ( 0.1 wt.\%) salt range. Hence, it is a demanding task to do the controlled filling of samples (of the order of $0.5 \mathrm{cc}$ ) for the light scattering experiments. It is found that phase transition is of first order all along except at one point. In other words, there exists a unique critical point. We have determined the coexistence boundary (visually) for a given $E / W$ ratio by varying the salt in the total mixture. By observing the equal volume separa- 
tion, critical concentration is identified and light scattering sample is made in pyrex cell with polished windows. The sample compostion in weight fraction is as follows: ethanol(0.211613); salt(0.138047); and water(0.650339). E/W ratio is $24.5505 / 75.4495$. It has the transition temperatures $289.36 \mathrm{~K}, 301.05 \mathrm{~K}$ and $336.661 \mathrm{~K}$, undergoing a sequence of these phase transitions: 2phase-1phase2 phase-1phase. The sole critical point is at $301.05 \mathrm{~K}$. We have measured the osmotic compressibility $\left(\chi_{T}\right)$ as one approaches this critical point from above and from below. Figure 3 presents the measurement of scattered intensity on approach to the transition point from above. It is a path of approach to the convex phase boundary. The details of the light scattering experiments remain the same as discussed in the previous section. With the aid of Eqs. (2) and (3), the measured scattered intensity $\left(I_{s}\right)$ at $90^{\circ}$, after correction, is fitted to the following expression without correction to scaling terms:

$$
\frac{I_{S}}{T}=\frac{A_{1} t^{-\gamma}}{1+A_{2} t^{-2 \nu}}
$$

where the reduced temperature $t$ is given in this case by $t=\left|\left(T_{U}-T\right) / T_{U}\right|$, where $\mathrm{T}_{U}$ is upper transition temperature. $\gamma$ and $\nu$ are critical exponents for osmotic compressibility $\left(\chi_{T}\right)$ and correlation length $\xi$ respectively and $A_{1}$ and $A_{2}$ are corresponding critical amplitudes. The fit of the experimental data to the above expression with respect to upper transition temperature $T_{U}$ is made(Fig.3). The experimental data were well described only by the mean-field exponent $(\gamma=1)$ which is demonstrated in the minimum $\chi_{\nu}{ }^{2}$ and the distribution of residuals, as shown in Fig.4.

The following best fit parameters were obtained: $\gamma=0.985, \mathrm{~T}_{U}=336.305, \chi_{\nu}{ }^{2}$ $0.333, \mathrm{~A}_{1}=(4.866 \pm 0.054) \times 10^{-2}$, and $\mathrm{A}_{2}=(2.832 \pm 0.883) \times 10^{-4}$. Correlation length amplitude $\left(\xi_{o}\right)=(8.91 \pm 5.78) \AA$. Attempt to fit Ising and correction-toscaling forms resulted in poorer fits, ruling out the relevance of Ising exponent $(\gamma=1.24)$ in this case.

\section{INVESTIGATIONS NEAR A PLAIT POINT}

An analogous system [ethanol + water + sodium carbonate] (to the one described in the preceding section) exhibits drastic change in the character of phase transitions. It did not show any reentrant behaviour throughout the experimental range (of temperature and concentrations of components) covered. In a ternary system, a critical point can be obtained at a given temperature by a suitable choice of concentration of the each component. This isothermal critical mixing point is called a Plait Point. We have determined various isothermal sections of the global coexistence surface. A section corresponding to $40^{\circ} \mathrm{C}$ is chosen for the measurement of osmotic compressibility $\left(\chi_{T}\right)$ as one approaches the Plait point. At plait point, in principle, a ternary system should show[1] the Fisher renormalised exponents $\alpha^{*}=-\alpha /(1-\alpha), \beta^{*}=\beta /(1-\alpha)$ and $\gamma^{*}=\gamma /(1-\alpha)$. Light scattering measurements were performed on approach to a plait point of $40^{\circ} \mathrm{C}$ in this system and data are shown in Fig. 5. The experimental details in this case are the same as discussed in the previous section. All Plait points in the temperature range covered $\left(26-70^{\circ} \mathrm{C}\right)$ have salt composition above $11 \%$ by weight. Analysis of the data of scattered intensity of light on approach to the Plait point shows that the exponent $(\gamma)$, describing the divergence of the $\chi_{T}$, is well described by a mean-field value $(\gamma=1)$, as supported by Figs. 5 and 6 . The results of the fit to Eq. (5) are $T_{c}=313.661 \mathrm{~K}, \gamma=1.00, \chi_{\nu}{ }^{2}=1.1505, \mathrm{~A}_{1}$ $=(431.231 \pm 2.065) \times 10^{-2}, \mathrm{~A}_{2}=(18.9045 \pm 2.8955) \times 10^{-5}$ and $\xi_{o}=(7.329$ 
土2.868) $\AA$. Attempt to fit the data to Ising and correctionto-scaling terms did not give satisfactory fit and physically meaningful parameter values. Though the system is non-ionic in nature, the critical exponent describing the divergence of $\chi_{T}$ exhibits mean-field value $(\gamma=$ 1 ). It is evident that the meanfield behaviour overrides and one could not observe the Fisher

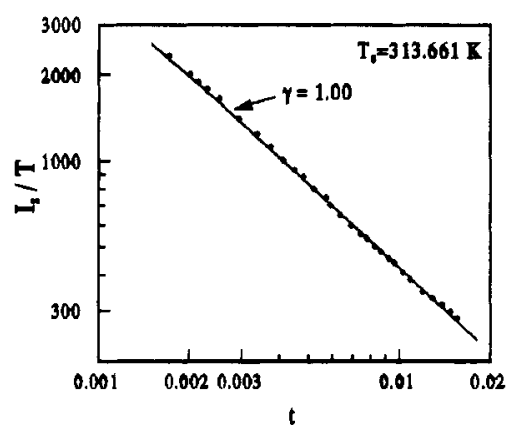

Fis. 5. Plot of acatrered intenulty ( $\mathrm{G} / \mathrm{T}$ ) versus distasce $t\left[=\left\langle\left(T_{c}-T\right) / T_{c}\right|\right]$ near the plait point $\left(T_{c}\right)$ for the ternary liquid mixtare [Ethanol + Water $\left.+\mathrm{Na}_{2} \mathrm{CO}_{3}\right]$. The solid liae is a fit to the experimeatal data points.

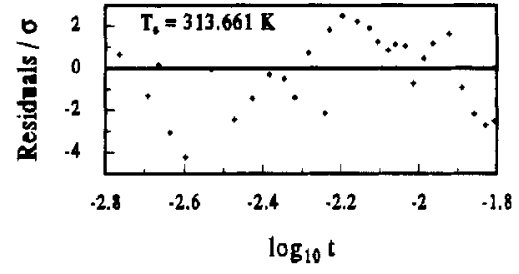

Fig. 6. Residuals of the fit to Eq. (5) for the data shown in Fig.5.

renormalization (because $\alpha$ has a mean-field value of 0 ). We attribute this finding to the structure promotion effects of the ionic solute.

\section{DISCUSSION}

We have obtained new evidence concerning the existence of crossover between Ising-like and Mean-field critical behaviour in an essentially solvophobic system - a ternary liquid mixture (3-methylpyridine + water + sodium bromide), as shown in Fig. 1. We anticipate that the crossover region will shift closer to the relevant critical point $\left(\mathrm{T}_{U}\right.$ or $\left.\mathrm{T}_{L}\right)$ as the amount of salt is increased in this system (beyond 8 weight \%). An open question in this problem, that is a matter of current debate[7], is the nature of crossover (i.e., monotonic or sharp) in terms of a universal exponent $\left(\gamma_{e f f}\right)$. The above crossover behaviour has been reported in metal - ammonia solutions[8], polymer blends[9] and ionic fluids[10].

The subtle relevance of the Mean-field critical behaviour (in the above system, Fig. 2) is more directly evident near a CIP [ in (ethanol + water + potassium carbonate), Fig. 3 ] and near a Plait point [in (ethanol + water + sodium carbonate), Fig. 5]. In fact, one anticipates only 3D Ising critical behaviour in all the three systems. A plausible explanation for the lack of Ising critical behaviour is the suppression of the growth of the concentration fluctuations (near the relevant phase transitions or $T_{c}$ ) by an additional length scale. This length scale can result from a possible structuring of the liquid mixtures around the ionic solute as is the case in metal - ammonia solutions and aqueous alcohols. Nevertheless, an independent and direct evidence of the structuring in these systems is desirable by these techniques: small angle X-ray scattering, small angle neutron scattering, partial molar volume measurements, hypersound measurements[11], etc. 


\section{ACKNOWLEDGEMENTS}

We are indebted to T.Narayanan for inspiring and fruitful discussions. We thank E.S.R.Gopal for his encouragement. We gratefully acknowledge the financial support for this research by the Department of Atomic Energy and the Department of Science and Technology.

\section{REFERENCES}

1. T. Narayanan and A. Kumar, Phys. Rep. 249, 135-218 (1994).

2. S. Venkatachalam, A. Kumar, and E.S.R. Gopal, J. Chem. Phys. 103, 6645-66 (1995).

3. T. Narayanan, A. Kumar, S. Venkatachalam, J. Jacob, and B. V. Prafulla, J. Chem. Phys. 102, 9653-9658 (1995).

4. G. M. Schneider, Ber. Bunsenges. Phys. Chem. 76, 325-331 (1972).

5. P. R. Bevington, 'Data Reduction and Error Analysis for the Physical Sciences', (McGraw Hill, New York, 1969).

6. J. P. Novak and G. M. Schneider, Ber. Bunsenges. Phys. Chem. 72, 791-798 (1968).

7. M. A. Anisimov, A. A. Povodyrev, V. D. Kulikov, and J. V. Sengers, Phys. Rev. Lett. 75, 3146-4095 (1995); see also C. Bagnuls and C. Bervillier, Phys. Rev. Lett. 76, 4094 (1996).

8. P. Chieux, J. Phys. (Paris) IV 1, C5-373 - C5-375 (1991).

9. F. S. Bates, J. H. Rosedale, P. Stepanek, T. P. Lodge, P. Wiltzius, G. H. Fredrickson, and R. P. Hjelm, Jr., Phys. Rev. Lett. 65, 1893-1896 (1990).

10. T. Narayanan and Kenneth S. Pitzer, J. Chem. Phys. 102, 8118- 8131 (1995).

11. S. V. Krivokhizha, I. L. Fabelinskii, L. L. Chaikov, and A. A. Shubin, JETP Lett. 64, 177-179 (1996). 